\title{
Revisão sistematizada das orientações sobre prevenção de distúrbios osteomusculares relacionados à Odontologia
}

\author{
Systematic review of guidances about prevention of musculoskeletal \\ disorders related to Dentistry \\ Revisión sistematizada de las directrices sobre la prevención de los \\ trastornos musculoesqueléticos relacionados con la Odontologia \\ Maria Luisa Gomes FERREIRA 1 \\ Marcos Sérgio ENDO2 \\ Mitsue FUJIMAKI ${ }^{2}$ \\ Luiz Fernando LOLLI ${ }^{2}$ \\ Najara Barbosa da ROCHA ${ }^{2}$ \\ ${ }^{1}$ Graduanda em Odontologia da Universidade Estadual de Maringá, UEM, Departamento de Odontologia, \\ 87083-170 Maringá - PR, Brasil \\ ${ }^{2}$ Docente do curso de Odontologia da Universidade Estadual de Maringá, UEM, Departamento de Odontologia, \\ 87083-170 Maringá - PR, Brasil
}

\section{Resumo}

Introdução: Os distúrbios osteomusculares relacionados ao trabalho são comuns nos cirurgiões-dentistas. Porém, ainda há uma carência de estudos e discussões acerca desse tema. Objetivo: Identificar fatores de risco, as formas comuns de distúrbios osteomusculares relacionados ao trabalho na área de Odontologia e prevenção. Métodos: Realizou-se uma revisão de literatura nas bases de dados Lilacs, Medline e Scielo utilizando os descritores: Transtornos Traumáticos Cumulativos, Doenças Profissionais e Odontologia. Os critérios de inclusão foram: estudos sobre prevenção e tratamento de distúrbios osteomusculares relacionados ao trabalho na odontologia; trabalhos completos e disponíveis; idioma português ou inglês; todo tipo de estudo e sem restrição quanto ao ano de publicação. Resultados: A pesquisa resultou 32 artigos. Após leitura dos títulos e resumos foram selecionados 11 artigos para análise na íntegra. Destes, 6 foram excluídos após aplicação dos critérios de exclusão, totalizando 4 publicações. Os fatores de risco apontados para o desenvolvimento dos distúrbios osteomusculares foram os movimentos repetitivos, falta de ergonomia, postura inadequada, força excessiva nos procedimentos, repouso insuficiente, pouco condicionamento físico e pressão psicológica. As formas comuns apontadas foram tendinites, tenossinovites, síndrome do túnel do carpo, miosites e bursites. O diagnóstico da doença no Cirurgião-Dentista é realizado pelo estudo da vida profissional, história da doença e exame físico, com tratamento interprofissional. Para prevenção os estudos destacaram o estilo de vida saudável, a prática de esportes, alongamento, controle do estresse, organização e adoção de princípios ergonômicos. Conclusão: Os cirurgiões-dentistas são propensos aos distúrbios osteomusculares relacionados ao trabalho, sendo necessária prevenção destas patologias desde a graduação, pois hábitos deletérios podem ser prolongados para a vida profissional.

Descritores: Transtornos Traumáticos Cumulativos; Doenças Profissionais; Programa de Prevenção de Riscos no Ambiente de Trabalho; Odontologia.

\section{Abstract}

Introduction: The work related musculoskeletal disorders (WRMSD) are common in dental surgeons. However, there is still a lack of studies and discussions on this topic. Objective: Identify the risk factors, the common forms of of work-related musculoskeletal disorders and their prevention in the area of Dentistry. Methods: A literature review was carried in the Lilacs, Medline and Scielo database out using descriptors: Cumulative trauma disorders, Occupational diseases and Dentistry,. The inclusion criteria were: studies on the prevention and treatment of work related musculoskeletal disorders in dentistry; complete and available Works; Portuguese or English language; all types of study and without restriction year of publication. Results: The search resulted in 32 articles. After read the titles and abstracts were selected 11 articles for analysis in full. Of these, 6 were excluded after applying the exclusion criteria, totaling 4 publications. The risk factors for the development of musculoskeletal disorders were repetitive movements, lack of ergonomics, inadequate posture, excessive force in procedures, insufficient rest, poor physical conditioning and psychological pressure. The common forms mentioned were tendinitis, tenosynovitis, carpal tunnel syndrome, myositis and bursitis. The diagnosis of the disease in the Dental Surgeon is performed by the study of the professional life, history of the disease and physical examination, with interprofessional treatment. For prevention the studies highlighted the healthy lifestyle, the practice of sports, stretching, stress control, organization and adoption of ergonomic principles. Conclusion: Dental surgeons are prone to work-related musculoskeletal disorders, and it is necessary to prevent these pathologies from graduation, since deleterious habits can be prolonged for professional life.

Descriptors: Cumulative Trauma Disorders; Occupational Diseases; Program of Risk Prevention on Working Environment; Dentistry.

\section{Resumen}

Introducción: Los trastornos musculoesqueléticos relacionados con el trabajo son comunes en los dentistas. Sin embargo, todavía hay escasez de estudios y debates sobre este tema. Objetivo: Identificar los factores de riesgo, las formas comunes de los trastornos musculoesqueléticos relacionados con el trabajo en el campo de la odontología y la prevención. Métodos: Se realizó una revisión bibliográfica en las bases de datos Lilacs, Medline y Scielo mediante los descriptores: Trastornos de traumas acumulados, Enfermedades profesionales y Odontología, Los criterios de inclusión fueron: estudios de prevención y tratamiento de los trastornos musculoesqueléticos relacionados con el trabajo en odontología; trabajos completos y disponibles; idioma portugués o inglés; todo tipo de estudio y año de publicación sin restricciones. Resultados: La búsqueda ha resultado 32 artículos. Después de la lectura de los títulos y resúmenes se seleccionaron 11 artículos para análisis en su totalidad. De estos, 6 fueron excluidos después de la aplicación de los criterios de exclusión, totalizando 4 publicaciones. Los factores de riesgo apuntados para el desarrollo de los trastornos osteomusculares fueron los movimientos repetitivos, falta de ergonomía, postura inadecuada, fuerza excesiva en los procedimientos, reposo insuficiente, poco condicionamiento físico y presión psicológica. Las formas comunes apuntadas fueron tendinitis, tenosinovites, síndrome del túnel carpiano, miositis y bursitis. El diagnóstico de la enfermedad en el Cirujano-Dentista es realizado por el estudio de la vida profesional, historia de la enfermedad y examen físico, con tratamiento interprofesional. Para prevenir los estudios destacaron el estilo de vida saludable, la práctica de deportes, estiramiento, control del estrés, organización y adopción de principios ergonómicos. Conclusión: Los cirujanos-dentistas son propensos a los disturbios osteomusculares relacionados al trabajo, siendo necesaria prevención de estas patologías desde la graduación, pues hábitos deletéreos pueden ser prolongados para la vida profesional.

Descriptores: Trastornos de Traumas Acumulados; Enfermedades Profesionales; Programa de Prevención de Riesgos en el Ambiente de Trabajo; Odontología.

\section{INTRODUÇÃO}

Os distúrbios osteomusculares relacionados ao trabalho (DORT) representam um problema de saúde muito prevalente no mundo atual, acometendo diversas categorias de trabalhadores. Os profissionais da Odontologia apresentam muitos desses processos patológicos que estão relacionadas diretamente ao ambiente de trabalho ${ }^{1}$.
São doenças derivadas de atividades profissionais, no qual o esforço repetitivo é uma constante. Durante a atividade profissional, há uma intensa solicitação muscular durante várias horas por dia ${ }^{1}$. Os distúrbios podem causar fadiga, falta de resistência, fraqueza, tremores, sensação de peso, falta de coordenação, dormência dos membros, dor ou irritação dos 
membros afetados, entorpecimento, formigamento ou perda de sensação, inabilidade ao manusear objetos, dificuldade ao abrir e/ou fechar as mãos, articulações enrijecidas, dores ou dormência nas mãos e punhos ao acordar e no decorrer da manhã, mãos frequentemente frias, necessidade de automassagem frequente, dificuldade ao executar movimentos precisos $^{2}$.

Os DORT podem ser controlados se diagnosticado no início da manifestação dos sintomas ${ }^{3}$. São doenças de difícil diagnóstico, pois dependem de diversos fatores como o relato subjetivo de quem as possui, aspectos psicológicos e a suscetibilidade individual. Portanto, a prevenção é de extrema importância a fim de evitar a doença, bem como a evolução para uma síndrome dolorosa crônica ${ }^{4}$.

A doença irá se manifestar em alterações que podem atingir músculos, fáscias musculares, vasos, tendões, ligamentos, nervos e articulações ${ }^{5}$. São caracterizados por uma dor crônica que atinge principalmente os dedos da mão, punho, antebraços, cotovelos, braços, ombros, pescoço, e regiões escapulares ${ }^{1}$. Eles não possuem causa única, havendo uma série de fatores que colaboram para o seu aparecimento, como movimentos repetitivos, o uso de aparelhos não ergonômicos, a postura de trabalho inadequada, o uso de força excessiva nos procedimentos, o repouso insuficiente, a falta de condicionamento físico, a pressão psicológica por resultados e as metas de produtividade, entre outros ${ }^{6}$.

Tendinites, bursites, miosites, síndrome do túnel do carpo e tenossinovites, são as formas mais comuns de DORT observados nos cirurgiões-dentistas ${ }^{1}$. Em relação ao tratamento para esses processos patológicos, destaca-se o trabalho multidisciplinar de profissionais de diversas áreas, a fim de reinserir o profissional no mercado de trabalho ${ }^{7}$.

$\mathrm{Na}$ literatura há poucos trabalhos relacionados aos distúrbios osteomusculares relacionados ao trabalho em Odontologia, bem como sobre a sua prevenção ${ }^{1,5}$. Contudo, os profissionais da área odontológica são bastante suscetíveis a essas patologias e há uma necessidade de informá-los e conscientizá-los. Por isso este trabalho objetivou realizar uma revisão de literatura crítica sobre DORT, a fim de sensibilização desses profissionais, principalmente os discentes, docentes, profissionais e técnicos sobre a instalação e prevenção destas doenças.

\section{MATERIAL E MÉTODO}

Realizou-se uma revisão de literatura, por dois pesquisadores independentes (NBR, MLGF), em duas fases, utilizando as palavras-chave: Transtornos Traumáticos Cumulativos, Doenças profissionais e Odontologia, na base de dados Lilacs, Medline e Scielo, de modo a identificar as publicações que continham orientações sobre a prevenção de distúrbios osteomusculares em Odontologia.

Os critérios de inclusão foram: estudos sobre a prevenção e tratamento de DORT na odontologia; trabalhos completos; idioma português ou inglês; todo tipo de estudo e sem restrição do ano de publicação do estudo. Os critérios de exclusão foram: artigos que não falavam especificamente de DORT, trabalhos incompletos e artigos que não continham texto completo disponível.

Para atingir os objetivos da discussão desta revisão, alguns temas foram explorados: tipos de DORT, fatores de risco, tratamento e forma de prevenção.

Os estudos foram levantados em duas fases: 1) resumos e títulos foram selecionados e 2) os textos completos dos títulos selecionados foram obtidos e lidos para determinar o conjunto de amostra final $(n=4)$. As etapas foram realizadas de forma independentes e reunião de consenso entre os artigos foram realizadas entre as etapas e, em caso a discordância permanecida, esta foi sanada por um terceiro pesquisador.

A síntese dos dados foi realizada por cada pesquisador que preencheu um formulário padronizado no programa Microsoft Excel, com os seguintes dados do artigo: autores, ano de publicação, objetivos, resultados e conclusão.

\section{RESULTADOS}

A pesquisa com os descritores selecionados resultou em 32 artigos nas bases de dados. Após verificação dos critérios de inclusão e exclusão, foram selecionados 11 artigos para análise na íntegra, sendo excluídos 6 artigos. O resultado final da revisão foram 4 publicações, sendo todos classificados como artigos científicos (Figura 1). As características dos estudos selecionados se encontram na Tabela 1

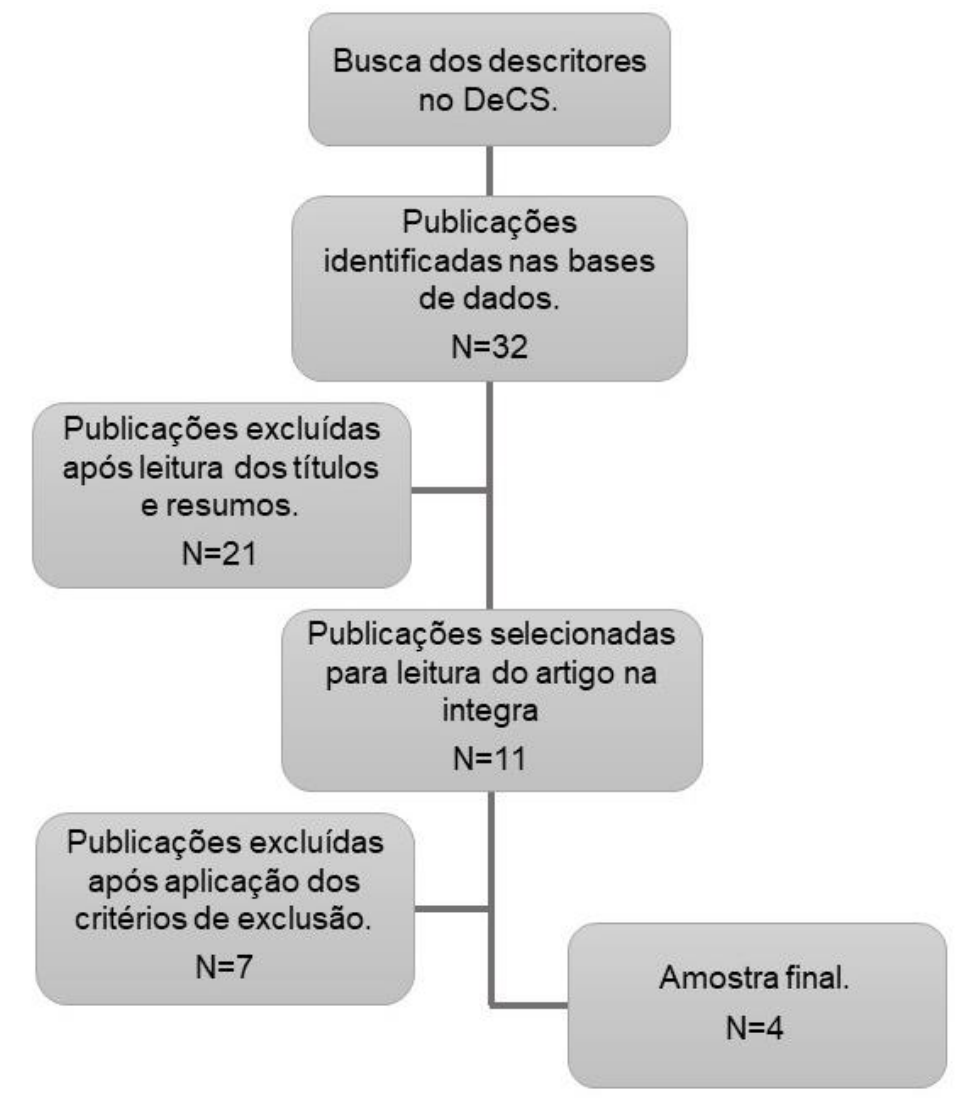

Figura 1: Fluxograma descritor do processo de seleção dos estudos para revisão.

Tabela 1 - Síntese dos artigos incluídos na revisão de acordo com os autores, ano, objetivo, DORT ou sintomas mais frequentes, prevenção, e resultados mais relevantes.

\begin{tabular}{|c|c|c|c|c|}
\hline $\begin{array}{l}\text { Autor } \\
\text { (ano) }\end{array}$ & Objetivo & $\begin{array}{l}\text { DORT ou } \\
\text { sintomas } \\
\text { mais } \\
\text { frequentes }\end{array}$ & Prevenção & $\begin{array}{l}\text { Resultados mais } \\
\text { relevantes }\end{array}$ \\
\hline $\begin{array}{l}\text { Coelho } \\
(2013)\end{array}$ & $\begin{array}{l}\text { Investigar as possíveis } \\
\text { lesões que acometem } \\
\text { os graduandos do } \\
\text { Curso de Odontologia } \\
\text { da Faculdade de } \\
\text { Odontologia de Bauru } \\
\text { (FOBUSP) e suas } \\
\text { relacốes com a } \\
\text { atividade laboral. }\end{array}$ & $\begin{array}{l}\text { Tendinite, } \\
\text { epicondilite } \\
\text { lateral, bursite, } \\
\text { síndrome do } \\
\text { túnel do carpo, } \\
\text { cisto sinovial. }\end{array}$ & $\begin{array}{c}\text { Educacãao } \\
\text { postural, } \\
\text { exercícios de } \\
\text { relaxamento e } \\
\text { alongamento na } \\
\text { rotina diária } \\
\text { (ginástica laboral), } \\
\text { realizacááde de } \\
\text { atividades físicas. }\end{array}$ & $\begin{array}{l}\text { A frequência dos } \\
\text { relatos de sintomas } \\
\text { dos DORT já está } \\
\text { presente nos alunos } \\
\text { de graduação e } \\
\text { parece aumentar } \\
\text { conforme o ano de } \\
\text { graduação. }\end{array}$ \\
\hline $\begin{array}{l}\text { Carvalho }{ }^{10} \\
\text { et al. }(2009)\end{array}$ & $\begin{array}{l}\text { Avaliar a presença de } \\
\text { distúrbios } \\
\text { osteomusculares } \\
\text { relacionados ao } \\
\text { trabalho entre } \\
\text { estudantes de } \\
\text { odontologia em duas } \\
\text { escolas odontológicas } \\
\text { brasileiras. }\end{array}$ & $\begin{array}{l}\text { A dor muscular } \\
\text { foi caracterizada } \\
\text { como o sintoma } \\
\text { mais comum. }\end{array}$ & $\begin{array}{l}\text { Postura de } \\
\text { trabalho correta, } \\
\text { utilização de } \\
\text { equipamentos } \\
\text { adequados e } \\
\text { ergonômicos, } \\
\text { téncicas de } \\
\text { relaxamento } \\
\text { muscular, prática } \\
\text { regular de } \\
\text { exercícios físicos. }\end{array}$ & $\begin{array}{c}\text { Os estudantes das } \\
\text { duas escolas de } \\
\text { odontologia no Brasil } \\
\text { relataram dor ou } \\
\text { fadiga durante ou logo } \\
\text { após o tratamento de } \\
\text { pacientes e foi } \\
\text { verificada a falta de } \\
\text { informação sobre } \\
\text { ergonomia no } \\
\text { ambiente acadêmico. }\end{array}$ \\
\hline $\begin{array}{l}\text { Samatoi }{ }^{11} \\
\text { et al. (2008) }\end{array}$ & $\begin{array}{l}\text { Determinar a } \\
\text { prevalênncia e as } \\
\text { associaçōes de } \\
\text { doenças devido a } \\
\text { sintomas a } \\
\text { musculoesqueléticos } \\
\text { entre cirurgióes } \\
\text { dentistas da Nova } \\
\text { Zelândia. }\end{array}$ & $\begin{array}{l}\text { Os sintomas } \\
\text { mais relatados } \\
\text { foram de dores } \\
\text { principalmente } \\
\text { no pescoço, } \\
\text { regiāo lombar e } \\
\text { ombros. }\end{array}$ & $\begin{array}{c}\text { Os cirurgiões } \\
\text { dentistas precisam } \\
\text { empregar postura } \\
\text { correta e } \\
\text { realização de } \\
\text { exercícios de } \\
\text { relaxamento para } \\
\text { minimizar o } \\
\text { estresse muscular } \\
\text { e esquelético. }\end{array}$ & $\begin{array}{l}\text { A prevalência de } \\
\text { sintomas } \\
\text { musculoesqueléticos } \\
\text { entre os cirurgioeses } \\
\text { dentitstas da Nova } \\
\text { Zelândia foi } \\
\text { considerada alta. }\end{array}$ \\
\hline $\begin{array}{l}\text { Alexopoulos } 12 \\
\text { et al. (2004) }\end{array}$ & $\begin{array}{l}\text { Investigar as relaçōes } \\
\text { entre características } \\
\text { físicas, , ssicossociais e } \\
\text { individuais das queeixas } \\
\text { musculoesqueléticas } \\
\text { de lombar, pescoco,o, } \\
\text { ombros e mão / pulso. }\end{array}$ & $\begin{array}{l}\text { Altas } \\
\text { prevalências de } \\
\text { dor nas costas, } \\
\text { pescoço, ombro } \\
\text { equeixas de } \\
\text { mão / pulso. }\end{array}$ & $\begin{array}{l}\text { Intervenções } \\
\text { ergonômicas }\end{array}$ & $\begin{array}{l}\text { Ocorrência de queixas } \\
\text { musculoesqueléticas } \\
\text { entre dentistas foi } \\
\text { associada à carga } \\
\text { física. Os fatores } \\
\text { psicossociais } \\
\text { relacionados ao } \\
\text { trabalho } \\
\text { desempenharam } \\
\text { papel inconsistente no } \\
\text { desenvolvimento da } \\
\text { cronicidade das } \\
\text { queixas. }\end{array}$ \\
\hline
\end{tabular}




\section{DISCUSSÃO}

Os resultados mostraram que os cirurgiões-dentistas são profissionais que apresentam um perfil predisponente para o desenvolvimento dos Distúrbios Osteomusculares Relacionados ao Trabalho ${ }^{5}$. Os fatores que estão presentes no cotidiano do profissional e que levam aos distúrbios são: esforço e força, postura inadequada, trabalho muscular estático, invariabilidade de tarefas, choques e impactos, pressão mecânica, vibração, frio, sobrecarga contínua das estruturas anatômicas ou falta de tempo para se recuperar no trabalho e fatores organizacionais. Sobre todos estes fatores ainda agem outros que seria a intensidade, a duração e a frequência ${ }^{1}$. Quanto maiores estes fatores, maior o risco ${ }^{8}$.

Por causa desta realidade, com a presença de fatores de riscos frequente no ambiente de trabalho, os profissionais da área de Odontologia estão muito suscetíveis ao desenvolvimento dos DORT, devendo estar atentos aos primeiros sinais de alteração para que possam prevenir e tratá-las o mais cedo possível, evitando o comprometimento de estruturas anatômicas importantes para a profissão, aumentando a longevidade laboral ${ }^{1}$.

Os artigos selecionados nesta revisão apontam estudos relacionados aos DORT na Odontologia. Infelizmente ainda há pouca literatura sobre o assunto especificamente. $\mathrm{O}$ estudo de Coelho ${ }^{9}$ consistiu em investigar as possíveis lesões que acometem os Graduandos do Curso de Odontologia da Faculdade de Odontologia de Bauru (FOBUSP) e suas relações com a atividade laboral, avaliando o impacto que as possíveis lesões causam na qualidade de vida dos alunos. $\mathrm{O}$ trabalho de Carvalho et al. ${ }^{10}$ realizou um estudo analítico transversal para avaliar a presença de DORT entre estudantes de Odontologia em duas universidades do Brasil. O estudo de Samatoi et al. ${ }^{11}$ objetivou determinar a prevalência de sintomas musculoesqueléticos entre os cirurgiões-dentistas de Nova Zelândia. O último artigo selecionado, de Alexopoulos et al. ${ }^{12}$, teve como objetivo investigar as relações entre as características físicas, psicossociais e individuais que podem levar as queixas de dores musculoesqueléticas principalmente de lombar, pescoço, ombro e mão/pulso.

Nos estudos, houve o relato dos DORT ou sintomas mais frequentes entre os profissionais da Odontologia. No artigo de Coelho $^{9}$ as doenças apresentadas com maior frequência foram tendinite, epicondilite lateral, bursite, síndrome do túnel do carpo e cisto sinovial'. No estudo de Carvalho et al. ${ }^{10}$ foi relatado como sintoma mais comum a dor muscular, principalmente nas regiões cervical, dorsal e lombar. Samatoi et al. ${ }^{11}$ citaram que os sintomas mais relatados foram dor no pescoço, região lombar e ombros. $\mathrm{O}$ estudo de Alexopoulos et al. ${ }^{12}$ relatou que $62 \%$ dos dentistas tiveram pelo menos uma queixa de dor musculoesquelética. Os sintomas mais frequentes foram dores nas costas, nos ombros e nas mãos/pulsos.

A prevenção é um fator muito importante para evitar o aparecimento de DORT, e apesar da adoção dessas medidas, podem surgir distúrbios se o ambiente de trabalho não estiver com formas ergonômicas de execução ${ }^{13}$. As ações preventivas dos DORT quando adotadas pelos profissionais irão propiciar uma melhora em sua qualidade de vida. Dessa forma, em relação à prevenção foi observada uma unanimidade entre os trabalhos analisados. As formas de prevenção que foram reiteradas nas pesquisas foram intervenções ergonômicas. De acordo com Gabin et al. ${ }^{14}$, a ergonomia nada mais é que um conjunto de saberes multidisciplinares que, quando são aplicados corretamente na organização das atividades laborativas e no posto de trabalho, apresenta como objetivo estabelecer um ambiente seguro, saudável e confortável, prevenindo os agravos à saúde contribuindo assim para sua eficiência produtiva.

A educação postural tem como finalidade possibilitar à pessoa ser capaz de se proteger ativamente em seus segmentos móveis de lesão dentro das condições de vida diária e profissional, seja no plano estático ou dinâmico. Dentro do campo de trabalho do cirurgião-dentista, com a finalidade de prevenção aos DORT, faz-se necessário a utilização de equipamentos adequados e ergonômicos ${ }^{15}$.

Para Puriene ${ }^{16}$ é fundamental que o dentista mantenha uma postura ergonomicamente correta no seu trabalho sentado, ou seja, deve sempre trabalhar com as costas relativamente retas e apoiadas no mocho. A permanência em uma postura inadequada, quando mantida por um longo período de tempo, durante toda uma jornada de trabalho é um dos principais fatores que predispõe a dor e desconforto do trabalhador, sendo a dor decorrente de um desequilíbrio osteomuscular que tende a ser localizada manifestando-se com maior frequência nas regiões da coluna cervical e lombar?.

A ginástica laboral é definida como um conjunto de exercícios físicos elaborados a partir da atividade profissional, que é realizada no próprio local de trabalho e durante o expediente de trabalho com duração média de 5 a 15 minutos, que se baseia na execução de exercícios de relaxamento, alongamento e resistência muscular de forma livre e espontânea, sem levar o trabalhador ao cansaço. Apresenta ação preventiva e terapêutica como no caso dos DORT, normalizando as capacidades e funções corporais para o desenvolvimento do trabalho, relaxando e tonificando as estruturas ${ }^{1}$.

Contudo, faz-se necessário que o profissional evite o sedentarismo e realize atividades físicas. Constatou-se que os DORT são considerados um dos maiores problemas da saúde ocupacional, na qual apresentam também fatores psicossociais que são tão importantes quanto os fatores físicos no desenvolvimento, exacerbação ou aceleração desses distúrbios ${ }^{9,17}$

O tratamento para DORT é realizado de forma multidisciplinar ${ }^{7}$, no qual os profissionais de diferentes áreas trabalham de forma integrada, associando diferentes recursos terapêuticos, desde o uso de medicação, fisioterapia, exercícios físicos e até bloqueios anestésicos e cirurgias nos casos mais avançados ${ }^{1}$. Terapias alternativas como acupuntura, homeopatia, laserterapia entre outras são bastante difundidas.

De acordo com os resultados mais relevantes das pesquisas, expostos na tabela 1 , pode-se perceber que 0 profissional da área odontológica apresenta uma alta predisposição para o acometimento dos DORT. No artigo de Carvalho et al. ${ }^{10}$ foi apresentado que $42 \%$ dos estudantes avaliados relataram que já haviam sido diagnosticados com DORT, como bursite e tendinite. Isso nos mostra que a propensão para os DORT está presente desde a graduação e tende a estender para a vida profissional. Assim, há necessidade de conscientização desses profissionais sobre os DORT, bem como as formas de prevenção já na graduação e isso deve ser enfatizado nos planos de ensino da graduação. A adoção de um estilo de vida saudável, a prática de esportes, a realização de exercícios de alongamento, medidas para controlar ou minimizar situações de estresse, organização no trabalho e adoção de princípios ergonômicos são fatores de proteção contra estas doenças.

Por ser muito difícil de obter um diagnóstico precoce, em função da ausência de sintomas iniciais, é muito importante evitar o surgimento dos DORT adotando sempre comportamentos e atitudes preventivas. Por outro lado, quando os casos são detectados no início tem mais chances de serem curados, assim é preciso identificar os riscos de desenvolver o distúrbio e eliminá-los de forma rápida, a medida do possível, mantendo sempre uma boa postura e não fazer força ou pressão exagerada ${ }^{9,18}$.

Os dados apresentados abrem novos questionamentos e direcionam para outros estudos nesta área. Há uma 
necessidade de realização de estudos longitudinais sobre este assunto, devido à escassez de dados a médio e longo prazo. Espera-se que este estudo proporcione subsídios para o planejamento e revisão de condutas e protocolos relativos à prevenção de DORT para os profissionais e para o ensino deste distúrbio já na graduação, bem como melhorar a orientação e conhecimento nesta área.

\section{CONCLUSÃO}

A partir dos resultados desta revisão crítica, sugere-se que os cirurgiões-dentistas são profissionais propensos aos DORT, desde a graduação, sendo necessária a prevenção destas doenças ocupacionais para melhorar a qualidade e longevidade de sua vida profissional o mais precocemente possível.

\section{REFERÊNCIAS}

1. Medeiros UV, Segatto GG. Lesões por esforços repetitivos (LER) e distúrbios osteomusculares (Dort) em dentistas. Rev bras odontol. 2012; 69(1):49-54.

2. Quilter D. The repetitive strain injury recovery book. New York: Walker; 1998.

3. Santos HH. Abordagem clínica e psicossocial das lesões por esforços repetitivos LER / DORT. Rev bras saúde ocup. 2003; 28(105-106):105-15.

4. Alencar MCB, Ota NH. O afastamento do trabalho por LER/DORT: repercussões na saúde mental. Rev Ter Ocup. 2011; 22(1):60-7.

5. Régis FGI, Michels G, Sell I. Lesões por esforços repetitivos/distúrbios osteomusculares relacionados ao trabalho em cirurgiões-dentistas: aspectos biomecânicos. Prod. 2009; 19(3):569-80.

6. Almeida CGSTG, Fernandes RCP. Distúrbios musculoesqueléticos em extremidades superiores distais entre homens e mulheres: resultados de estudo na indústria. Rev Bras Saude Ocup. 2017; 42:e3.

7. Ikari TE, Mantelli M Corrêa Filho HR, Monteiro MI. Tratamento de LER/Dort: intervenções fisioterápicas. Rev ciênc méd. 2007; 16(4/6): 233-43.

8. BRASIL. Ministério da Saúde. Departamento de Ações Programáticas e Estratégicas. Área Técnica de Saúde do Trabalhador. Lesões por esforços repetitivos (LER) - e Distúrbios Osteomusculares Relacionados ao Trabalho (Dort). Brasília: Ministério da Saúde; 2001.

9. Coelho TRF. Sintomas de DORT de acadêmicos de odontologia: uma contribuição para prevenção com ginástica laboral. Dissertação [Mestrado em Ciências Odontológicas Aplicadas]. Bauru: Faculdade de Odontologia de Bauru / USP; 2013.

10. Carvalho MVD, Soriano EP, Caldas AF, Campello RIC, Miranda HF, Cavalcanti FIV. Work-related musculoskeletal disorders among brazilian dental students. J Dent Educ. 2009; 73(5):624-30.

11. Samatoi A, Moffat SM, Thonson WN. Musculoskeletal symptoms in New Zealand dental therapists: prevalence and associated disability. New Zealand Dent J. 2008; 104(2): 49-5.

12. Alexopoulus EC, Stathi IC, Charizani F. Prevalence of musculoskeletal disorders in dentists. BMC Musculoskelet Disord. 2004; 5:16.

13. Vilela RAG, Almeida IM, Mendes RWB. Da vigilância para prevenção de acidentes de trabalho: contribuição da ergonomia da atividade. Ciênc Saúde Colet. 2012; 17(10): 2817-2830.

14. Garbin AJI, Garbin CAS, Diniz DG. Normas e diretrizes ergonômicas em odontologia: o caminho para a adoção de uma postura de trabalho saudável. Rev Odont Univ São Paulo 2009; 21(2): 155-161.

15. Braccialli LMP, Vilarta R. Aspectos a serem considerados na elaboração de programa de prevenção e orientação de problemas posturais. Rev paul Educ Fís. 2000; 14(2):159-71.

16. Puriene A, Aleksejuniene J, Petrauskiene J, Balciuniene I, Janulyete V. Self-Reported Ocupacional Health issues among Lithuanian dentists. Industrial Health. 2008; 46(4):369-74.

17. Araújo MA, Paula MVQ. LER/DORT: um grave problema de saúde pública que acomete os cirurgiões dentistas. Rev APS. 2003; 6(2):87-93.

18. Trindade E, Andrade M. LER/DORT: Rotina Dolorosa. Rev ABO Nac. 2003; 11(2):72-5.

\section{CONFLITO DE INTERESSES}

Os autores declaram não haver conflitos de interesse.

\section{AUTOR PARA CORRESPONDÊNCIA}

\section{Maria Luísa Gomes Ferreira}

malu.odt@gmail.com
Submetido em 08/03/2018 Aceito em 09/04/2018 